\title{
DISCOVERY OF A DOUBLE RING IN THE DWARF GALAXY MARKARIAN 409
}

\author{
A. Gil de Paz, ${ }^{1}$ B. F. Madore, ${ }^{1,2}$ K. Noeske, ${ }^{3}$ L. M. Cairós, ${ }^{3}$ P. Papaderos, ${ }^{3}$ and S. A. Silich ${ }^{4}$ \\ Received 2003 July 18; accepted 2003 September 5; published 2003 September 30
}

\begin{abstract}
We report the discovery of a double ring of emission-line regions around the nucleus of the blue compact dwarf galaxy Mrk 409 as seen by deep, ground-based H $\alpha$ images. Echelle spectroscopy obtained at Magellan I with MIKE shows the presence of ionized gas flowing out of the galaxy from a very massive $\left(\geq 7 \times 10^{6} M_{\odot}\right)$ nuclear starburst with projected expansion velocities of $\sim 50 \mathrm{~km} \mathrm{~s}^{-1}$. Different scenarios for the formation of these rings are discussed. While the innermost, nuclear ring is most probably formed by the interaction of a starburst-driven shock with the surrounding interstellar medium, the origin of the outer ring is less clear.
\end{abstract}

Subject headings: galaxies: dwarf — galaxies: evolution — galaxies: individual (Markarian 409, NGC 3011) — galaxies: kinematics and dynamics — galaxies: starburst

\section{INTRODUCTION}

In the present era of precision cosmology, where the fundamental parameters $\left(H_{0}, \Omega_{M}\right.$, and $\left.\Omega_{\Lambda}\right)$ have been measured with unprecedented accuracy (Bennett et al. 2003), an understanding of the evolution of baryonic matter becomes even more important than in the past. In this sense, the feedback of gas and metals collectively ejected by starburst galaxies is currently the least understood ingredient of state-of-the-art hierarchical models of galaxy formation and evolution (Kay et al. 2002; Somerville \& Primack 1999). This is particularly important in the case of dwarf galaxies because of their shallow gravitational potentials. Despite recent progress, the actual efficiency of this mechanism is still unknown, mainly because of our poor understanding of the initial, preburst galaxy state (Silich \& Tenorio-Tagle 2001; Mac Low \& Ferrara 1999).

In this Letter, we report the discovery of an unusual system of two concentric rings of ionized gas around a massive nuclear starburst in Mrk 409. Echelle spectroscopy obtained with MIKE at the $6.5 \mathrm{~m}$ Magellan I telescope at Las Campanas (Chile) shows ionized gas flowing out of the disk of the galaxy. These results lead us to suggest that the peculiar morphology of this system may be the result of the interaction of a collective starburst-driven shock with the galaxy's interstellar medium (ISM). Understanding the formation of these peculiar structures may provide fundamental clues for the study of the evolution of starbursts and their impact on the future evolution of dwarf galaxies.

Mrk 409 is a nearby blue compact dwarf (BCD) galaxy $\left(v_{\text {helio }}=1527 \mathrm{~km} \mathrm{~s}^{-1}\right.$ ) with a $B$-band luminosity $M_{B}=$ $-17.27 \pm 0.03$ ( $h=0.7$; see Gil de Paz, Madore, \& Pevunova 2003, hereafter GMP). It has been recently included in the Nearby Field Galaxy Survey of Jansen et al. (2000), and it was identified as a possible counterrotator by Kannappan \& Fabricant (2001, hereafter KF01).

\footnotetext{
${ }^{1}$ Carnegie Observatories, Carnegie Institution of Washington, 813 Santa Barbara Street, Pasadena, CA 91101-1292; agpaz@ociw.edu, barry@ociw.edu.

${ }^{2}$ NASA/IPAC Extragalactic Database, California Institute of Technology, MS 100-22, 770 South Wilson Avenue, Pasadena, CA 91125.

${ }^{3}$ Universitäts-Sternwarte Göttingen, Geismarlandstrasse 11, D-37083 Göttingen, Germany; knoeske@uni-sw.gwdg.de, luzma@uni-sw.gwdg.de, papade@uni-sw.gwdg.de.

${ }^{4}$ Instituto Nacional de Astrofísica, Óptica y Electrónica, AP 51, Luis Enrique Erro 1, Tonantzintla 72000, Puebla, Mexico; silich@inaoep.mx.
}

\section{OBSERVATIONS AND REDUCTION}

On 2002 May 14-15, we obtained optical $B R$ and narrowband $\mathrm{H} \alpha(\Delta \lambda \simeq 20 \AA)$ images of Mrk 409 at the Palomar 60 inch telescope using a $2048 \times 2048 \mathrm{CCD}$. Additional $B R$ images were taken with the 1.23 and $2.2 \mathrm{~m}$ telescopes at Calar Alto (Almería, Spain) on 2000 February 4 and 1997 March 10, respectively. Deeper, higher quality $R$ and $\mathrm{H} \alpha(\Delta \lambda \simeq 50 \AA)$ images were later obtained (2003 January 9) using the ALFOSC camera at the $2.5 \mathrm{~m}$ NOT telescope (La Palma, Spain), again using a $2048 \times 2048$ CCD. Exposure times were $960 \mathrm{~s}(R)$ and $7200 \mathrm{~s}(\mathrm{H} \alpha)$, and the seeing was in the range of 0 ".9-1".0. Finally, near-infrared $(J H K)$ images were obtained at the $1.5 \mathrm{~m}$ Carlos Sánchez telescope (Tenerife, Spain) on 2000 March 8 using the CAIN camera with exposure times of $960 \mathrm{~s}(J), 1140 \mathrm{~s}(H)$, and $1440 \mathrm{~s}(K)$. Images were reduced using standard procedures of bias subtraction and flat-fielding within IRAF. Figure 1 shows the different images obtained and the corresponding surface brightness and color profiles.

An echelle spectrum of Mrk 409 was obtained using MIKE at the Magellan I (Baade) telescope on Las Campanas (Chile) on 2003 April 28. For a complete description of MIKE, see Bernstein et al. (2003). The $1^{\prime \prime}$ wide ( $5^{\prime \prime} \mathrm{long}$ ) slit used provided a spectral resolution of $\sim 14.1 \pm 0.4 \mathrm{~km} \mathrm{~s}^{-1}$ (FWHM) in the light of $\mathrm{H} \alpha$. The position angle of the slit was $39^{\circ}$, and the exposure time was $1200 \mathrm{~s}$ (see Fig. 1b). The reduction and extraction of the spectrum were carried out using the IRAF task DOECSLIT. In Figure 2, we show the bidimensional image in the region of $\mathrm{H} \alpha$ (Fig. $2 a$ ) and the extracted spectrum in some of the lines detected (Figs. $2 b-2 f$ ).

\section{RESULTS AND DISCUSSION}

\subsection{Nuclear Starburst}

The center of Mrk 409 is of high surface brightness in the optical $\left(\mu_{B}=19 \mathrm{mag} \operatorname{arcsec}{ }^{-2}\right.$; see Fig. $\left.1 e\right)$. This value is 2 mag brighter than the value obtained from the extrapolation of the surface brightness profile of the underlying stellar population (21.16 mag $\operatorname{arcsec}^{-2}$ ), which dominates the profile in the outer regions of the galaxy. The enhancement in its surface brightness profile along with the presence of ionized gas emission associated with the galaxy nucleus indicate the presence of a nuclear starburst.

If we assume an exponential profile for the low surface brightness (LSB) underlying the stellar population, the decomposition of the surface brightness profile yields a total lumi- 

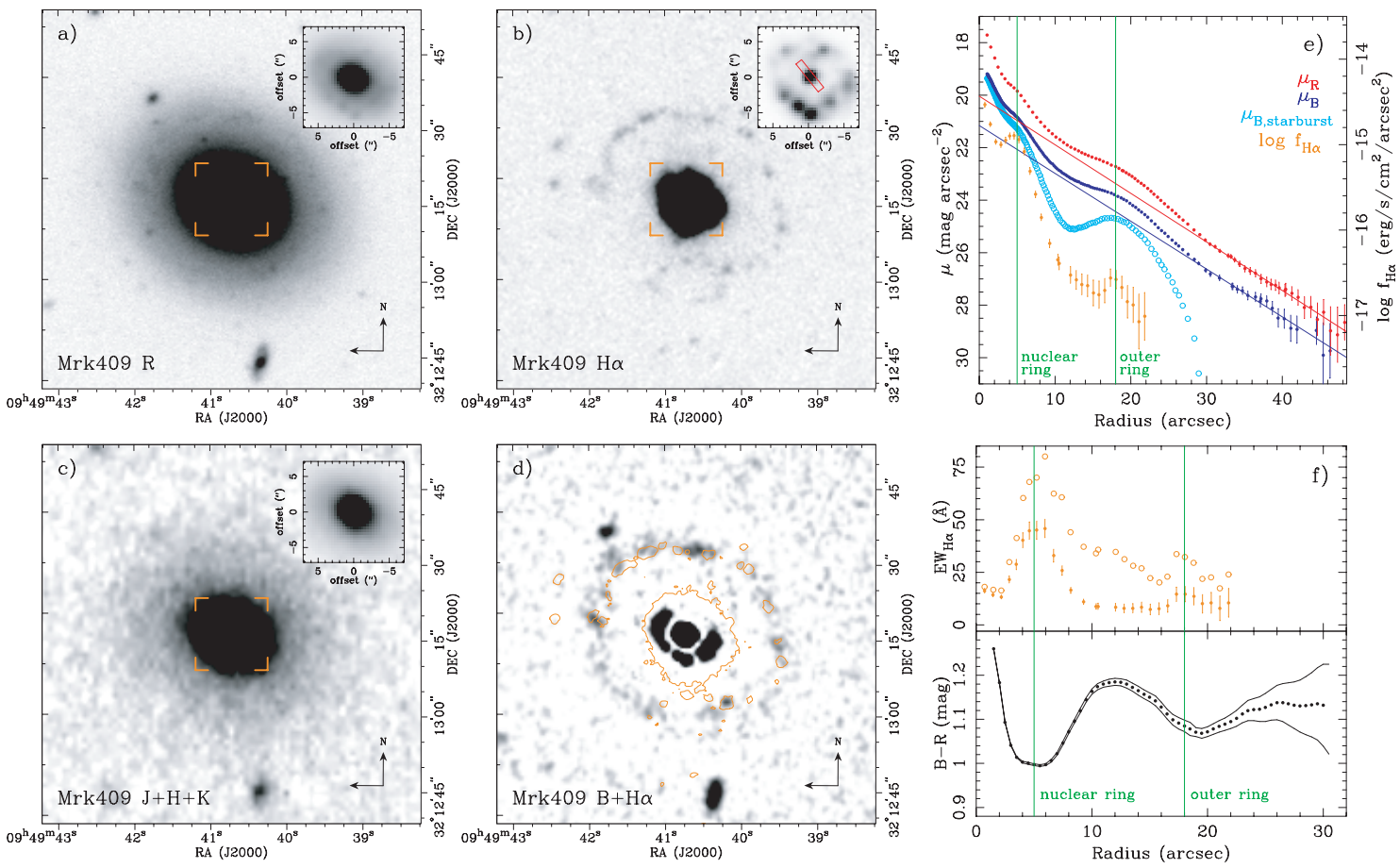

FIG. 1.-(a) $R$-band image of Mrk 409. An expanded view of the nuclear region is shown on the upper right-hand corner of each panel. (b) Continuum-subtracted $\mathrm{H} \alpha$ image. The image has been smoothed using a $2 \times 2$ pixels boxcar filter. The position of the slit used for our MIKE observations is shown in the upper righthand box. (c) $J+H+K$ image. (d) Unsharp-masked $B$-band image and $\mathrm{H} \alpha$ contours. (e) $B R$ and $\mathrm{H} \alpha$ surface brightness profiles. The open circles represent the starburst $B$-band surface brightness subtracted from the contribution of the LSB component. $(f) \mathrm{EW}(\mathrm{H} \alpha)$ (upper panel) and $B-R$ color (lower panel) profiles. The filled and open circles in the upper panel represent the starburst $\mathrm{EW}(\mathrm{H} \alpha)$ before and after the correction from the LSB component contamination, respectively.

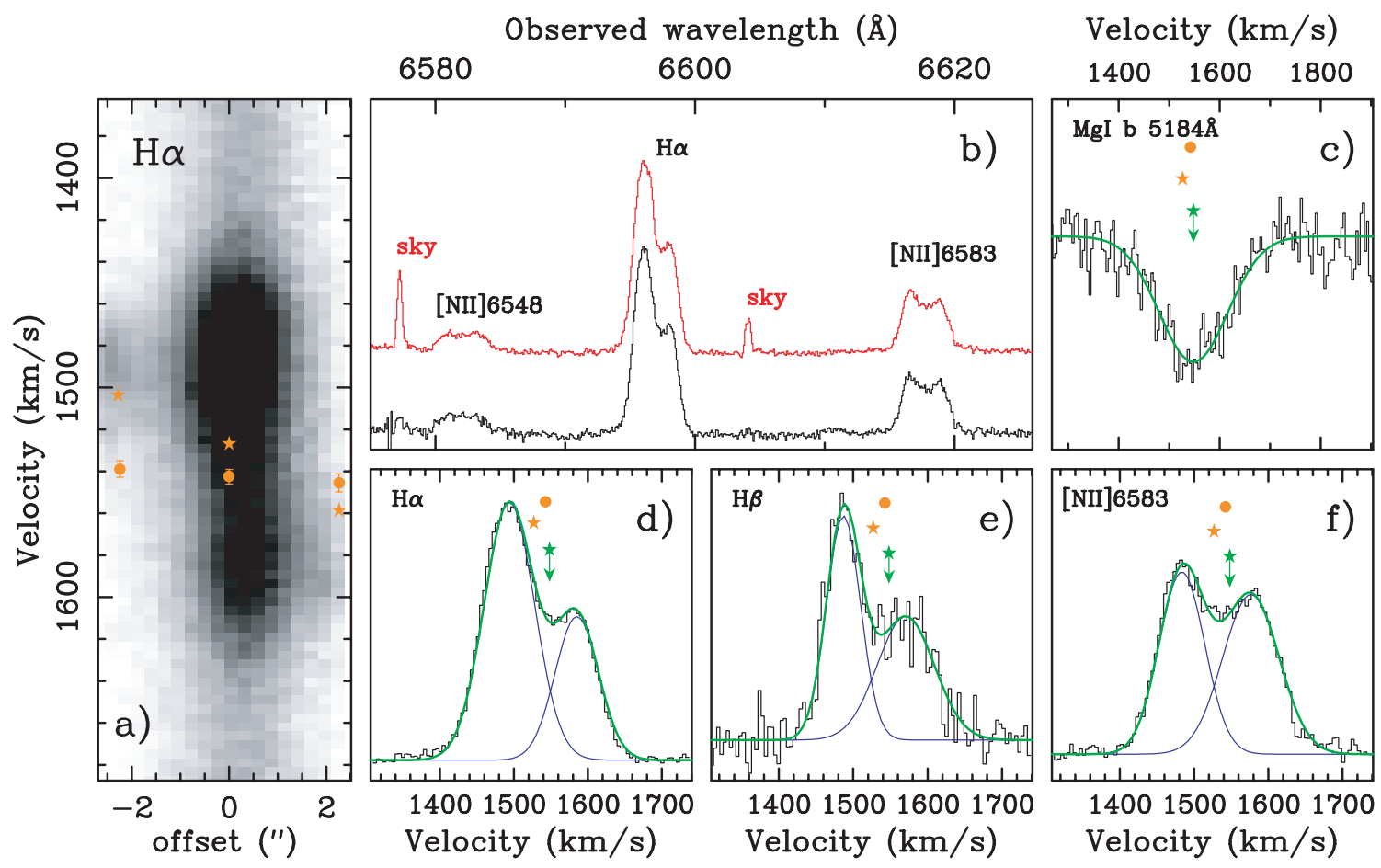

FIG. 2.-(a) Echelle spectrum of Mrk 409 in the $\mathrm{H} \alpha$ region obtained with MIKE at Magellan I. The positions and velocities for the gas (filled circles) and stars (star symbols) published by KF01 are shown. (b) Extracted spectrum in the region of $[\mathrm{N}$ II] $\lambda 6548, \mathrm{H} \alpha$, and $[\mathrm{N}$ II] $\lambda 6583$ with (red line) and without the sky (black line). (c) Extracted spectrum in the region of Mg I $b \lambda 5184$. The green star marks the best-fit stellar velocity. (d) Blowup in the H $\alpha$ line region. The bestfitting solution using the sum of two Gaussians is shown. (e) $\mathrm{H} \beta$. $(f)[\mathrm{N} \mathrm{II}] \lambda 6583$. 
nosity for the starburst of $M_{B}=-16.5 \mathrm{mag}$. However, the slightly convex shape of the surface brightness profile at distances greater than $28^{\prime \prime}$ suggests a central intensity flattening of the LSB component. Such flattening has recently been found to be common within BCDs (see Noeske et al. 2003). This fact along with the moderately high extinction present in the galaxy nuclear regions (inferred from the galaxy nuclear $B-R$ color and its Balmer decrement; see below and see also Jansen et al. 2000) make the number given above a lower limit to the starburst luminosity.

The echelle spectrum of the galaxy nucleus reveals the presence of a double-peaked $\mathrm{H} \alpha$ emission line with a total equivalent width of $11 \pm 1 \AA$ (see Fig. $2 b$ ). These two peaks are shifted by $-54 \pm 3$ and $37 \pm 3 \mathrm{~km} \mathrm{~s}^{-1}$ with respect to the radial velocity of the stars. The latter was obtained by fitting the profile of the $\mathrm{Mg}$ I $b \lambda 5184$ absorption line (see Fig. $2 c$ ).

Taking into account the contribution of the underlying stellar population and the corresponding uncertainties, the $\mathrm{H} \alpha$ equivalent width of the nuclear starburst should be in the range of 11-15 A. If we now assume an instantaneous burst with a Salpeter initial mass function $\left(1-100 M_{\odot}\right)$ and a typical metallicity of $Z_{\odot} / 5$, the age derived would be $\sim 10.7-11.3 \mathrm{Myr}$ (Leitherer et al. 1999). However, as we show below, a significant fraction of this emission is certainly arising from shockexcited gas. Therefore, since the timescale for the injection of mechanical energy is much longer than the photoionization timescale $(\sim 10 \mathrm{Myr})$, the nuclear starburst could be significantly older than this value. Thus, using $10 \mathrm{Myr}$ as a lower limit for the age, we derive a total stellar mass for the starburst of $\geq 7 \times 10^{6} M_{\odot}$.

The H $\alpha$ blue- and redshifted components show very different intensities. The approaching side is almost a factor of 2 brighter than the receding one. This cannot be attributed just to a dustopacity effect since the $\mathrm{H} \alpha / \mathrm{H} \beta$ ratio of the redshifted (4.86 \pm $0.25)$ and blueshifted $(4.91 \pm 0.25)$ components are comparable. The two components also show different excitation conditions. The $[\mathrm{N} \mathrm{II}] \lambda 6583 / \mathrm{H} \alpha$ line ratio of the redshifted component is $[\mathrm{N} \mathrm{II}] / \mathrm{H} \alpha_{\text {red }}=0.480 \pm 0.007$, while for the blueshifted component, it is $[\mathrm{N} \mathrm{II}] / \mathrm{H} \alpha_{\text {blue }}=0.306 \pm 0.005$ (see Figs. $2 d$ and $2 f)$. The $[\mathrm{S} \mathrm{II}] \lambda \lambda 6717,6731 / \mathrm{H} \alpha$ and $[\mathrm{O} \mathrm{III}] / \mathrm{H} \beta$ line ratios are $\log [\mathrm{S} \mathrm{II}] / \mathrm{H} \alpha_{\text {blue }}=-0.34$ and $\log [\mathrm{O} \mathrm{III}] / \mathrm{H} \beta_{\text {blue }}=0.51$, respectively, and $\log [\mathrm{S}$ II $] / \mathrm{H} \alpha_{\text {red }}=-0.12$ and $\log [\mathrm{O}$ III $] / \mathrm{H} \beta_{\text {red }}=$ 0.67 . These numbers indicate that the emission of both components, but particularly that of the redshifted one, has a significant contribution from shock-excited gas (see Martin 1997). One possible explanation is that the larger contribution from photoionized gas to the blueshifted component is due to its greater proximity to the ionization source. This would also explain why this component is brighter than the one arising from the more distant, shock-excited, redshifted gas. However, if that is the case, we should expect the blueshifted lobe to be moving with a lower velocity (with respect to the systemic velocity) than the redshifted one, in contradiction to what we observe.

It is also possible that either the amount of ionizing photons absorbed by dust before ionization or the escape fraction of these photons is significantly smaller for the blueshifted lobe than for the redshifted one. This would result both in a larger contribution from the photoionized gas emission than from the shock-excited gas and a larger total emissivity of the blueshifted lobe.

\subsection{Nuclear Ring}

At a distance of $5^{\prime \prime}(0.6 \mathrm{kpc})$ from the galaxy nucleus, we find an $\mathrm{H} \alpha$-emitting ring delineated by at least eight individual
$\mathrm{H}$ II regions (see Fig. 1b). The mean $\mathrm{H} \alpha$ equivalent width of the regions in the ring as measured from the azimuthally averaged $R$ and $\mathrm{H} \alpha$ surface brightness profiles is $45 \pm 4 \AA$. The equivalent width of $\mathrm{H} \alpha$ corrected for the contribution of the underlying stellar population is estimated to be in the range of 70-80 A (see Fig. 1f). Unfortunately, the continuum emission at the position of this nuclear ring is also strongly contaminated by the nuclear starburst, so $\operatorname{EW}(\mathrm{H} \alpha)$ values higher than this number are expected. With regard to the $B-R$ color, the position of the ring coincides with the bluest portion of the galaxy profile. Higher resolution images would be desirable in order to derive the properties (color, luminosity, age, and mass) of each individual region in the ring.

Interestingly, the distribution and properties of the regions in the nuclear ring resemble those in the BCD Mrk 86. Like in Mrk 409, Mrk 86 shows a ring of young $\mathrm{H}$ II regions at a galactocentric radius of $0.5-1 \mathrm{kpc}$ around a massive, relatively evolved nuclear starburst. In Gil de Paz, Zamorano, \& Gallego (2000a) and Gil de Paz et al. (2000b), we proposed a scenario for the evolution of Mrk 86 in which the gas, swept out by the nuclear starburst in the galactic plane, eventually reached densities large enough to become molecular, thereafter forming a ring of $\mathrm{H}$ II regions and newly born stars. The similarity in the apparent properties of these two galaxies suggests that the same mechanism could have taken place in Mrk 409.

Using our numerical model for the evolution of a starburstdriven bubble (Silich \& Tenorio-Tagle 1998) along with the evolution of the kinetic energy deposition rate predicted by the Leitherer et al. (1999) models for a $7 \times 10^{6} M_{\odot}$ massive starburst, we derive an average density for the surrounding ISM of $n_{\text {ISM }} \simeq 2 \mathrm{~cm}^{-3}$ and an evolutionary time of $\sim 7.4$ Myr. Here we have adopted a shell radius of $600 \mathrm{pc}$ and an expansion velocity of $50 \mathrm{~km} \mathrm{~s}^{-1}$. The small difference between the bubble evolutionary time and the starburst age can be explained as the time required for the coherent shell to form (see Silich et al. 2002).

The total $\mathrm{H} \alpha$ luminosity of the shell (considering both photo- and shock-ionized emission) can be written as $1.36\left(4 \pi R_{\text {shell }} n_{\mathrm{ISM}} v_{\text {shell }}+N_{\mathrm{UV}}\right) \times 10^{-12} \mathrm{ergs} \mathrm{s}^{-1}$, where $N_{\mathrm{UV}}$ is the number of ionizing photons per second escaping from the star cluster region. Adopting an evolutionary time for the bubble of 7.4 Myr and the number of Lyman photons emitted by the starburst as the value for $N_{\mathrm{UV}}$, the expected $\mathrm{H} \alpha$ luminosity would be $2.5 \times 10^{40} \mathrm{ergs} \mathrm{s}^{-1}$. This number is larger than the total $\mathrm{H} \alpha$ luminosity of the galaxy $\left(1.6 \times 10^{40} \mathrm{ergs} \mathrm{s}^{-1}\right.$; GMP), suggesting that a significant fraction of the ionizing photons is trapped within the nuclear starburst region, as it is also shown by Figure 1e, and/or that the actual starburst age exceeds the adopted value.

It is worth mentioning that according to the simulations carried out in Gil de Paz et al. (2002), the ring of swept-out gas is not necessarily in equilibrium and, in fact, has a rotational velocity smaller than that of the underlying stellar population. This behavior could explain the smaller velocity gradient of the gas as compared with the stars observed by KF01 in the inner regions of Mrk 409.

\subsection{Outer Ring}

Our deep H $\alpha$ image of Mrk 409 also reveals the presence of an outer ring of line-emitting clumps at a larger galactocentric distance (see Fig. 1b). A small enhancement in the continuum light is also observed in the broadband images at a similar position (see Figs. $1 d$ and 1e). The overall distribution of the brightest clumps in both the $\mathrm{H} \alpha$ image and the unsharp- 
masked $B$-band image seems to be well reproduced by an ellipse with a ellipticity of $\sim 0.1-0.2$, a position angle of $\sim 50^{\circ}$, and a major-axis radius of $19^{\prime \prime}(\sim 2 \mathrm{kpc})$. Note that although the rings in Mrk 409 are named according to the terminology used for lenticular and spiral galaxies, their formation mechanism may be different. In particular, Mrk 409 does not show any obvious oval distortion or bar, and these are believed to be responsible for the formation of rings in those galaxies (see Buta \& Combes 1996).

A first explanation for the geometry observed in $\mathrm{H} \alpha$ is that the outer ring represents the brightened limb of an hourglasslike structure of ionized gas with the innermost ring being the narrowest part of it, where the gas present in the galactic plane would have been swept out (see Silich \& Tenorio-Tagle 1998). However, both the enhancement in the continuum light associated with this ring (which suggests the presence of secondary star formation) and the high-intensity contrast between the ring and the interring regions allow us to rule out this scenario.

Therefore, in the case of the outer ring, an independent mechanism must have been responsible for shaping this ring and triggering its recent star formation activity. Some scenarios are proposed: (1) the existence of episodic starburst activity in the nucleus that has resulted in the formation, first, of the outer ring and, more recently, of the nuclear ring, (2) the merging with a companion galaxy or cloud, or (3) gas accretion from the galaxy halo.

The standard model equations for the evolution of the bubble radius and expansion velocity (Mac Low \& McCray 1988) imply that in order for scenario 1 to be compatible with the starburst mass and outer-ring radius measured, the host galaxy has to have an extended gaseous halo with an average density well below $1 \mathrm{~cm}^{-3}$. On the other hand, the remarkably regular morphology of the galaxy LSB component suggests that if scenario 2 is correct, the collision probably involved a very low mass gas-rich companion galaxy, like those proposed by Bergvall \& Östlin (2002) to explain the recent star formation activity in BCD galaxies.

Finally, scenario 3 requires that the outer-ring radius roughly coincides with the boundary of the radial velocity curve that shows solid-body rotation. However, stellar spectroscopy car- ried out by KF01 indicates that the radius where the rotation curve flattens $(\leq 0.7 \mathrm{kpc})$ is significantly smaller than the outerring radius $(\sim 2 \mathrm{kpc})$.

\section{CONCLUSIONS}

We have discovered a double ring of $\mathrm{H} \alpha$-emitting regions in the BCD Mrk 409. Although multiple rings are common in lenticular and spiral galaxies (Buta \& Combes 1996), this is the first time that such a complex morphology is observed in a dwarf galaxy like Mrk 409. Echelle spectroscopy reveals the presence of gas flowing out of the galaxy most probably associated with a starburst-driven supershell arising from a massive (relatively obscured) nuclear starburst. The formation of the nuclear ring is interpreted as being due to the sweeping of the ISM by the shock in the galactic plane.

The origin of the outer ring is less clear. Different scenarios are proposed: (1) the existence of previous nuclear starburst activity that shaped an expanding ring in a way similar to how the nuclear ring has been proposed to be formed, (2) the merging with a low-mass gas-rich companion galaxy, and (3) gas accretion from the galaxy halo.

In the future, we plan to obtain echelle spectra of individual clumps in the rings, which, in combination with our hydrodynamical models for the evolution of starbursts (Silich \& Tenorio-Tagle 1998), will allow us to understand the mechanisms that led to the formation of the structures observed in Mrk 409. This may provide further clues to understanding the actual impact of the recent star formation activity on the future evolution of dwarf galaxies.

We acknowledge the referee (R. Buta) for his valuable comments. We thank the staff at the Palomar, LCO, Teide, NOT, and CAHA observatories for their help and support. This work has been financed by the Spanish PN de Astronomía y Astrofísica grants AYA2000-1790 and AYA2003-1676, the DFG grants FR325/50-1 and FR325/50-2, the CONACYT (Mexico) grant 36132-E, the EC Marie-Curie grant HPMF-CT-200000774, and the NASA Galaxy Evolution Explorer mission.

\section{REFERENCES}

Bennett, C. L., et al. 2003, ApJS, 148, 1 (astro-ph/0302207)

Bergvall, N., \& Östlin, G. 2002, A\&A, 390, 891

Bernstein, R., Shectman, S. A., Gunnels, S. M., Mochnacki, S., \& Athey, A. E. 2003, Proc. SPIE, 4841, 1694

Buta, R., \& Combes, F. 1996, Fund. Cosmic Phys., 17, 95

Gil de Paz, A., Madore, B. F., \& Pevunova, O. 2003, ApJS, 147, 29 (GMP)

Gil de Paz, A., Silich, S. A., Madore, B. F., S anchez Contreras, C., Zamorano, J., \& Gallego, J. 2002, ApJ, 573, L101

Gil de Paz, A., Zamorano, J., \& Gallego, J. 2000a, A\&A, 361, 465

Gil de Paz, A., Zamorano, J., Gallego, J., Domínguez, F. de B. 2000b, A\&AS, 145,377

Jansen, R. A., Fabricant, D., Franx, M., \& Caldwell, N. 2000, ApJS, 126, 331

Kannappan, S. J., \& Fabricant, D. G. 2001, AJ, 121, 140 (KF01)
Kay, S. T., Pearce, F. R., Frenk, C. S., \& Jenkins, A. 2002, MNRAS, 330, 113

Leitherer, C., et al. 1999, ApJS, 123, 3

Mac Low, M.-M., \& Ferrara, A. 1999, ApJ, 513, 142

Mac Low, M.-M., \& McCray, R. 1988, ApJ, 324, 776

Martin, C. L. 1997, ApJ, 491, 561

Noeske, K. G., Papaderos, P., Cairós, L. M., \& Fricke, K. J. 2003, A\&A, in press

Silich, S. A., \& Tenorio-Tagle, G. 1998, MNRAS, 299, 249 2001, ApJ, 552, 91

Silich, S. A., Tenorio-Tagle, G., Muñoz-Tuñon, C., \& Cairós, L. M. 2002, AJ, 123,2438

Somerville, R. S., \& Primack, J. R. 1999, MNRAS, 310, 1087 